\title{
RESONANCIA MAGNÉTICA, HACIA LA CAPTURA DE LOS TIEMPOS FISIOLÓGICOS
}

TM. Iván Soza G.

Departamento de Imágenes

Servicio de Resonancia Magnética

Clínica Alemana de Santiago

email:isoza@alemana.cl

\section{RESUMEN}

Las tecnologías incorporadas en el último tiempo en resonancia magnética, han impactado considerablemente en la resolución temporal y espacial. Los avances más relevantes son; tecnología multicanal, adquisición paralela y bobinas multireceptoras.

La incorporación y asimilación de estas nuevas herramientas permite optimizar la calidad y tiempos de exámenes, respondiendo a las exigencias diagnósticas y asistenciales de esta técnica por imágenes.

Interiorizarse de la tecnología que se dispone y evaluar en que medida se puede participar de estos avances, permitirá trabajar con un máximo de rendimiento, para cada centro en particular.

\section{INTRODUCCIÓN}

Sin duda, la resonancia magnética (RM) ha evolucionando vertiginosamente durante los últimos años, siendo la carrera por la captura de los tiempos fisiológicos lo que impulsa el constante desarrollo de software, hardware, secuencias y herramientas que aceleran los procesos involucrados en la generación de la imagen. Este desafío es enfrentado con investigaciones y avances en tres grandes polos de desarrollo de la RM: transmisión, recepción y procesamiento de datos (Figura 1).

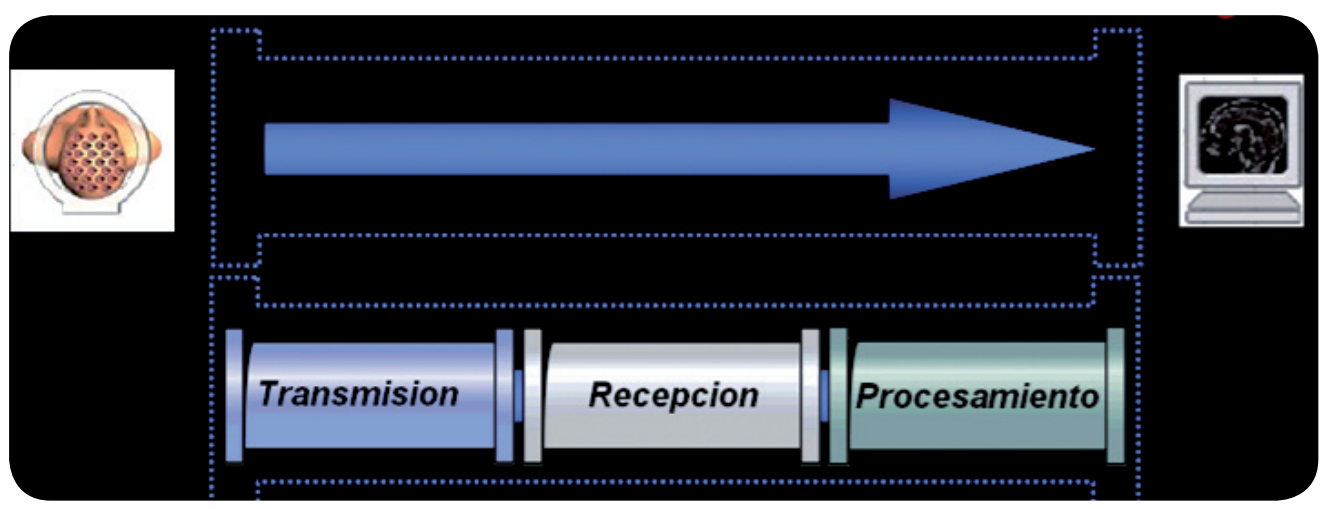

Figura 1. Transmisión relacionada con emisión de radiofrecuencia (RF). Recepción implica el desarrollo de nuevas bobinas. Procesamiento es la incorporación de computadores capaces de trabajar con una gran cantidad de información en tiempo corto. 
Basándonos en estos conceptos fundamentales, en el presente artículo describiremos los avances más importantes del último tiempo en RM, que han tenido un impacto relevante en la resolución espacial y temporal de las imágenes, que ha incidido en una mejoría de la sensibilidad y especificidad de los exámenes. Estos avances son los siguientes:

- Adquisición paralela

- Bobinas multireceptoras

- Equipos multicanales

\section{EVOLUCIÓN DE LA RM EN EL ÚLTIMO TIEMPO}

Desde que en 1971 Paul Lautenbeur planteó la idea de superponer un nuevo campo magnético (gradiente magnética) al campo principal, entregó al mundo de la RM la llave y los cimientos para la localización de la señal(1).

Los objetivos posteriores siempre confluyen en cómo aprovechar durante el proceso de relajación del hidrógeno una mayor recolección de señal en menos tiempo.

Poder responder hoy en día a todas las exigencias de las distintas áreas de la medicina, implica que las imágenes por RM deben entregar no sólo información anatómica sino que también en el campo funcional, donde las expectativas y aportes son hoy en día las de mayor interés.

El legado dejado por Peter Mansfield en 1977 permite abordar estos desafíos con las secuencias eco planar (EPI), que cambiaron el concepto de tren de ecos, reemplazando las RF por gradientes bipolares y digitalizando la señal en ambos sentidos del espacio K, impactando y llevando los tiempos de adquisición a los milisegundos.

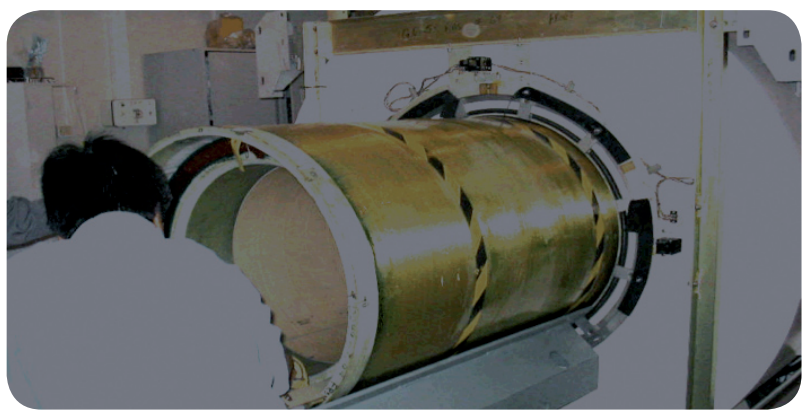

Figura 2. La bobina de gradientes, tiene un peso aproximado de $500 \mathrm{~kg}$. Contiene en su arquitectura los tres pares de bobinas $X, Y, Z$.

En pleno período de la resonancia moderna, comienza un desenfrenado y focalizado interés por el desarrollo de las bobinas de gradientes, llevando al máximo las exigencias y rendimiento de éstas. Este esfuerzo de los investigadores y de la industria se ha reflejado en la comercialización de nuevas generaciones de equipos que han permitido satisfacer las expectativas clínicas, aumentando y mejorando las aplicaciones del método (Figura 2).

Este desarrollo tecnológico de las bobinas de gradientes, no obstante, por razones de bioseguridad, es restringido por la FDA (Food and drug administration, EEUU) ${ }^{(2)}$, dictaminando límites de performance para el uso clínico. Estos límites regulan los riesgos biológicos de las gradientes de campo magnético, tales como la estimulación nerviosa periférica y ruido provocado por éstas, siendo el trauma acústico el problema mayor.

Así, la resonancia magnética se ve nuevamente enfrentando grandes desafíos, como seguir acortando los tiempos de exámenes, mejorando la resolución espacial y temporal con uno de sus principales componentes por el momento restringido en su máxima escalada.

En el año 2001 se comercializa la adquisición paralela, la cual fue desarrollada por Klaas P. Pruessmann, por lo que recibió el reconocimiento de la European Society for Magnetic Resonante Imaging el año 2004.

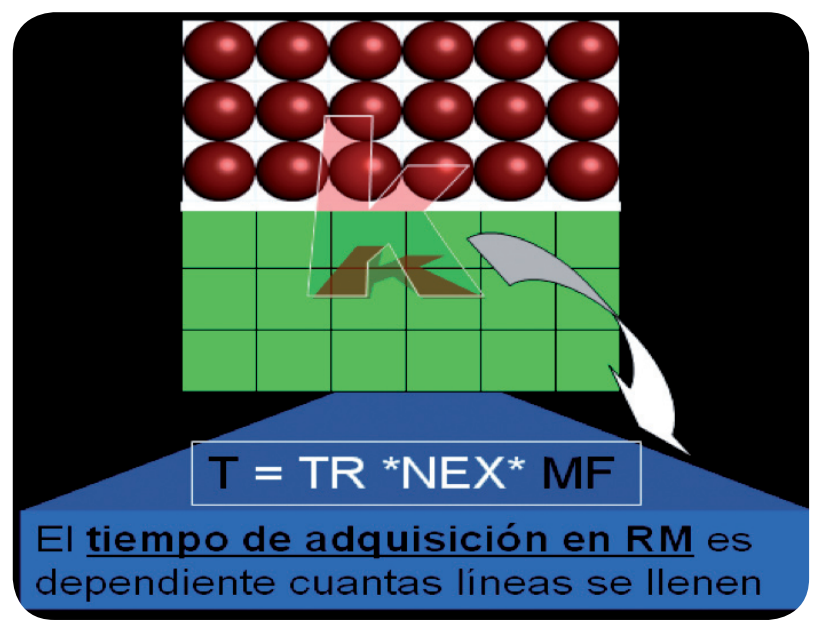

Figura 3. En la ecuación básica de tiempo de adquisición (T) en RM, cada línea de adquisición en el sentido de la matriz fase (MF) contribuye a aumentar el tiempo total de la secuencia.

Pruessmann, planteó bajar las líneas de codificación de fase sin alterar la resolución espacial. Para el pensamiento lógico de quienes participamos en esta técnica y partimos aprendiendo que cada línea de codificación de fase será un aporte en la resolución espacial, esto resulta ser muy revolucionario. Recordemos que las líneas de codificación de fase tienen una directa relación con los tiempos de adquisición (Figura 3 ).

De acuerdo a este planteamiento, si se llenan menos líneas de codificación de fase en el espacio $\mathrm{K}$, se reducen considerablemente los tiempos de adquisición de las secuencias.

Por lo tanto, lo que realizó Pruessmann fue reemplazar codificaciones de fase por procesos 
matemáticos que son los encargados de mantener la resolución espacial a posteriori con información previa de los receptores (elementos, bobinas).

\section{BOBINAS MULTIRECEPTORAS}

Para lograr este objetivo ya existía el concepto de multibobinas o multireceptores ${ }^{(3)}$ las cuales se agrupan de acuerdo a las necesidades del examen y en donde cada receptor obtiene una data particular aportando a una imagen total, esto es lo que llamamos dependiendo de las empresas comerciales bobinas Phased Array o Sinergy (Figura 4).

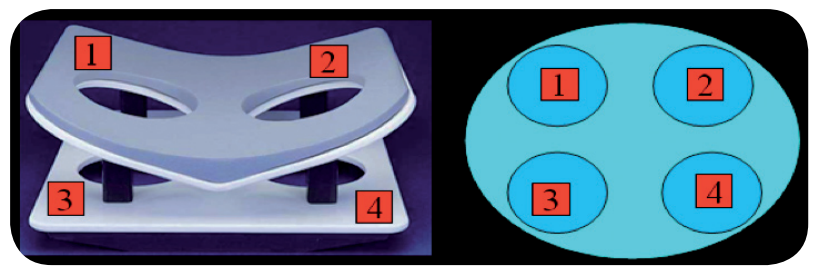

Figura 4. La bobina representada es una phased array, de cuatro receptores (elementos), cada receptor recibe señal independiente y luego contribuyen los cuatro a una imagen final, obteniendo mayor relación señal ruido y mayor cobertura.

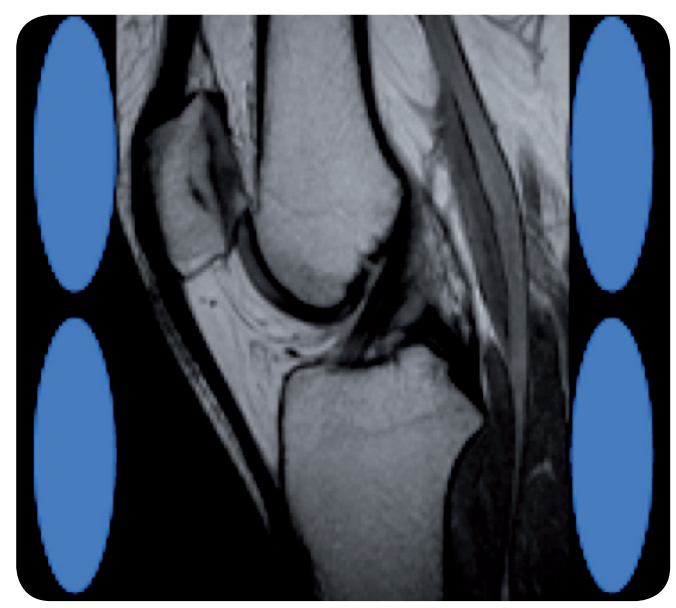

Figura 5. Cuatro receptores totales, dos anteriores y dos posteriores. Con esta condicionante, se puede realizar adquisición paralela.
Para realizar adquisición paralela este concepto de agrupar ("arreglo de bobinas") se mantiene ${ }^{(4)}$. La condicionante se origina a partir de que estos receptores deben estar dispuestos de tal manera que la estructura a estudiar debe tener receptores en paralelo respecto a la estructura (Figura 5).

Para poder realizar los procesos matemáticos involucrados en la reposición de las líneas del espacio $\mathrm{K}$ que son dejadas de adquirir, necesitamos entregar una data previa al sistema, esta se obtiene a partir de los perfiles de sensibilidad de los receptores. Estos perfiles de sensibilidad dependiendo de la empresa comercial se pueden adquirir antes o durante la secuencia de adquisición y son conocidos como calibración ${ }^{(5)}$, o reference scan. Estos datos son un muestreo rápido para cada receptor respecto a la anatomía que está enfrentando. Cuando los datos de los receptores son insuficientes, los perfiles de sensibilidad estarán incompletos y esto originará una gran cantidad de artefactos de adquisición paralela.

\section{ADQUISICIÓN PARALELA}

Con el fin de entregar una clasificación de acuerdo a los nombres asignados por las distintas empresas a la adquisición paralela, identifiquemos en primera instancia los procesos más relevantes en la RM: (Figura 6).

La adquisición paralela se puede dividir en dos grupos:

- Los procesos realizados después de la transformada de Fourier, en la imagen.

- Los procesos realizados en el espacio K.

\section{A. ADQUISICIÓN PARALELA REALIZADA DESPUÉS DE LA TRANSFORMADA DE FOURIER}

De acuerdo a las empresa comerciales reciben los siguientes nombres:

- SENSE (Sensitivy Encoding) Philips.

- ASSET (Array Spatial Sensitivy Encoding Technique) GE

- m SENSE (Self-calibrate SENSE) Siemens

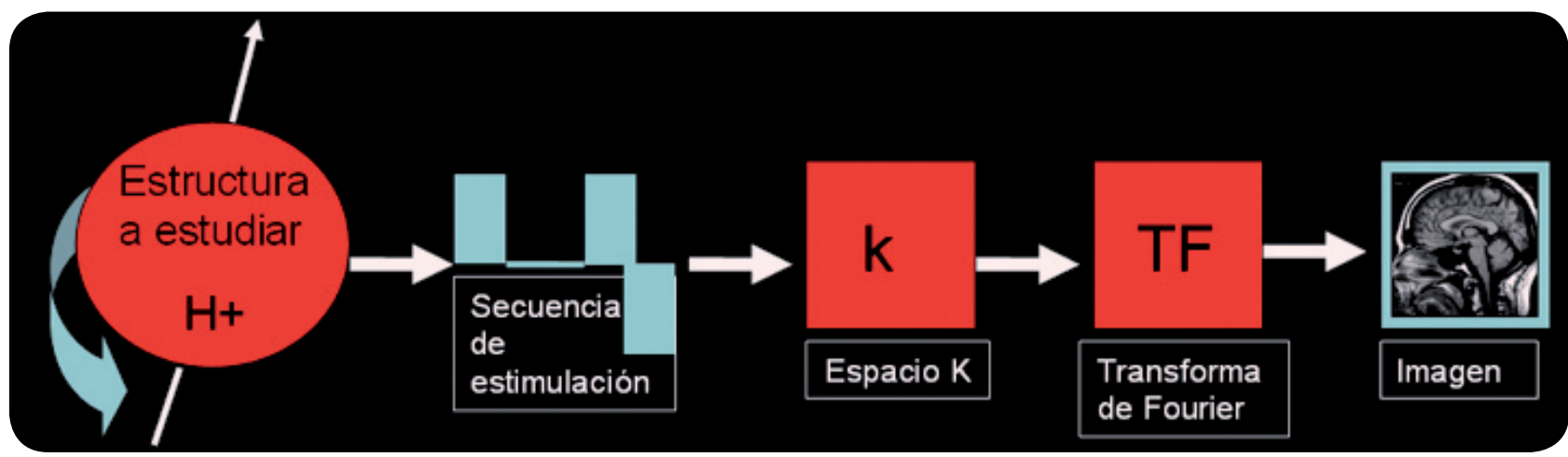

Figura 6. Secuencia básica, considerando población de hidrógeno, Secuencias de adquisición, datos al espacio K, transformada de Fourier, obteniendo luego la imagen. 
La adquisición paralela se crea con la finalidad de acortar los tiempos de adquisición en RM, favoreciendo de acuerdo a las necesidades diagnósticas del examen, la resolución temporal o espacial. Recordemos que la finalidad es no llenar la totalidad de las líneas de codificación de fase del espacio $\mathrm{K}$, de esta forma ya tenemos menos tiempo de adquisición (Figura 3). Siempre hay que tener presente que el uso de la adquisición paralela implica una caída de un $30 \%$ a $40 \%$ en la relación señal/ruido, por lo tanto no todas las secuencias con adquisición paralela obtienen imágenes óptimas y es en este momento cuando una bobina con mayor cantidad de receptores puede levantar la señal perdida, manteniendo el tiempo corto ya conseguido. Cuando se obtienen menos líneas del espacio K (Figura 7), se produce un "enrollamiento" de la imagen (Figura 8). Para poder "desenrollar" esta imagen recurriremos a los perfiles de sensibilidad de los receptores, los cuales ya contienen la data para restituir estas líneas y mantener la resolución espacial; todo esto sucede en el dominio de la imagen donde la resonancia ya cobró el tiempo de adquisición, el que fue más corto por haber llenado menos líneas en el espacio K.

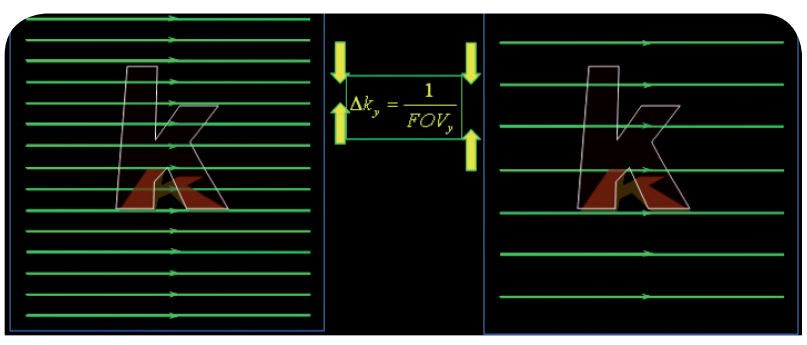

Figura 7. (A) Totalidad de las líneas del espacio K adquiridas. (B) Menor cantidad de líneas adquiridas.

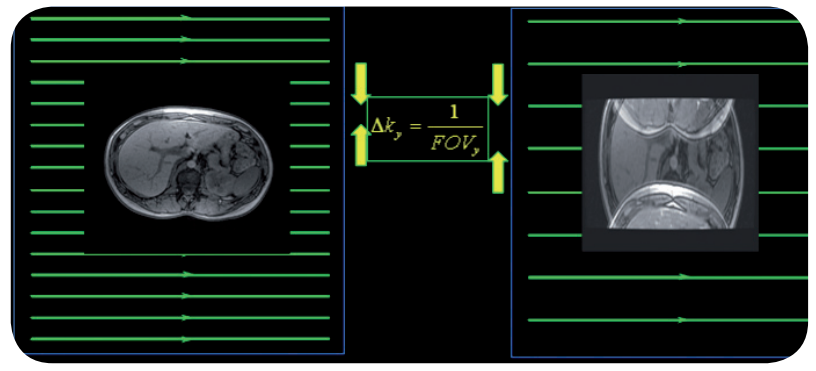

Figura 8. (A) Imagen completa con todas las líneas del espacio $\mathrm{K}$ adquiridas. (B) Imagen con enrollamiento con menos líneas del espacio $\mathrm{K}$ adquiridas.

Es importante siempre considerar que en la medida que llenamos menos líneas del espacio K, bajan los tiempos de adquisición. Hoy en día, el poder acelerar la velocidad de adquisición de una secuencia depende además de las características particulares de cada marca. De acuerdo a cada empresa comercial los equipos tendrán mayor o menor capacidad de aceleración, incluso esto se maneja como un dato de evaluación de equipos en las instancias de compra, si bien hay que distinguir entre la capacidad téorica de aceleración y la real, dada por la configuración del sistema como un todo. Esta capacidad de acelerar las secuencias, se describe en la consola como factor de aceleración o "R ". Si el valor de R es mayor, más rápida es la secuencia y menos líneas del espacio $\mathrm{K}$ son adquiridas, lo cual conlleva una mayor caída en la relación señal/ruido y mayor posibilidad de artefactos inducidos por adquisición paralela (Figura 9).

\section{B. ADQUISICIÓN PARALELA REALIZADA EN EL ESPACIO K}

La adquisición paralela realizada en el espacio $\mathrm{K}$ de acuerdo a las empresas comerciales reciben los siguientes nombres:

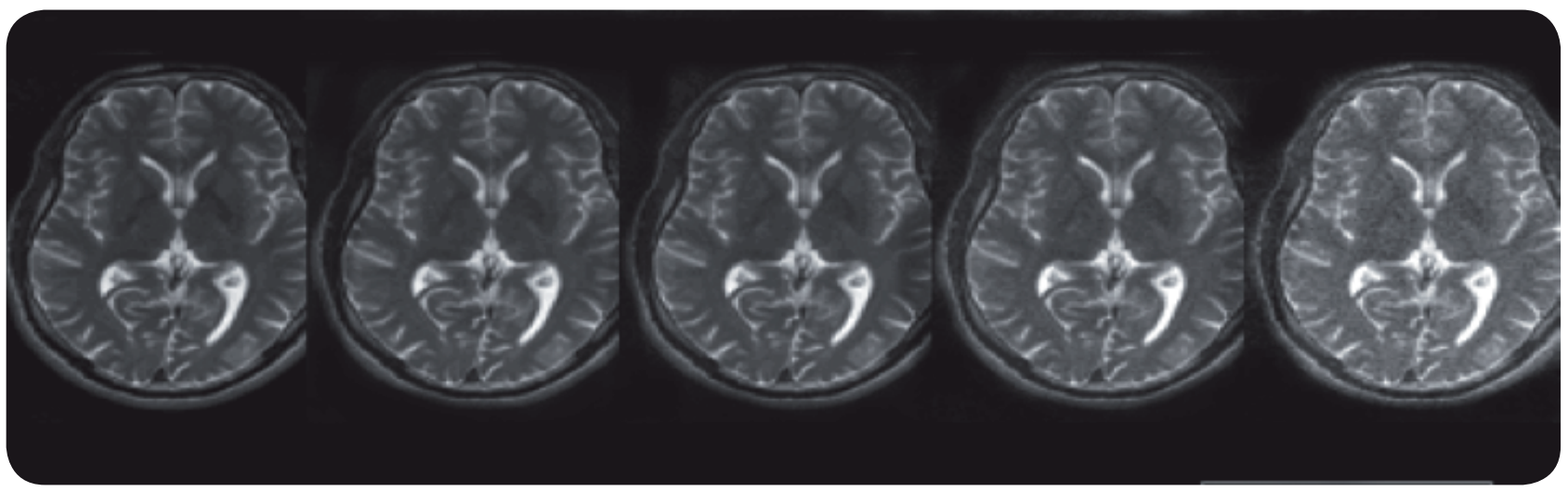

Figura 9. Imagen axial ponderada en T2 de cerebro con distintos factores de aceleración. Se observa que a medida que más velocidad se aplica a la secuencia hay un deterioro considerable en la imagen. 
- SMASH (Simultaneus Adquisition of Spatial Harmonics) ${ }^{(6)}$.

- GRAPPA (GeneRealized Autocalibrating Partially Parallel Acquisition) Siemens ${ }^{(7)}$.

Una de las grandes ventajas de esta técnica está relacionada con la ausencia de artefactos por enrollamiento, debido a que la adquisición paralela y los perfiles de sensibilidad actúan en el espacio K, antes de la generación de la imagen.

\section{EQUIPOS MULTICANAL}

A los conceptos de bobinas multireceptoras y adquisición paralela, simultáneamente los equipos comienzan a identificarse por su cantidad de canales.

La asimilación de estos conceptos ha sido difícil por lo rápido del cambio.

Cuando hacemos referencia a canales en RM nos referimos a la relación directa con las vías de transmisión de datos de los receptores de las bobinas hacia los computadores de procesamiento, por lo tanto siempre han existido los canales en los equipos de RM, sólo que no se hablaba de ellos, debido a que durante largo tiempo existieron cuatro vías de transmisión (canales) y la mayor cantidad de centros adquirió equipos con estas características. En este período se realizaron una gran cantidad de avances en RM, como difusión, perfusión, funcional, angiografías, imágenes cardíacas, exámenes con resolución temporal (abdomen, mamas) y espectroscopía.

Cuando se introduce la adquisición paralela y bobinas multireceptoras, esto conlleva a construir equipos con más vías de transmisión (más canales) y de esta forma se puede cumplir el objetivo final que es acortar los tiempos de adquisición.

Al construir bobinas con mayor cantidad de receptores, se plantea que cada receptor tenga un canal de transmisión (Figura 10), Si un equipo tiene más receptores y menos canales, este no trabajará en forma óptima porque se produce un cuello de botella en la vía de transmisión (Figura 11), por lo tanto un sistema ideal para un equipo, tiene que tener tantos canales como receptores (elementos) que demanden las bobinas mutireceptoras (multielementos) (Figura 12).

Es interesante destacar que la tecnología de canales para equipos de resonancia magnética va adelantada con respecto a la construcción de bobinas multireceptoras existentes en el mercado, por ejemplo, los equipos con capacidad de 16 ó 32 canales han desarrollado recién sólo una bobina con esta cantidad de receptores y la mayoría está aún en 8 ó 12 receptores como máximo.

Lo más importante para que el sistema funcione óptimamente es que tiene que existir un canal de transmisión para cada receptor. Ahora, cuando la bobina tiene más receptores que canales en el equipo, se ocupa el concepto ya mencionado, el cual es agrupar o usar un arreglo de receptores (concepto phased array o sinergy), adecuándose a esta condición (Figura 13).

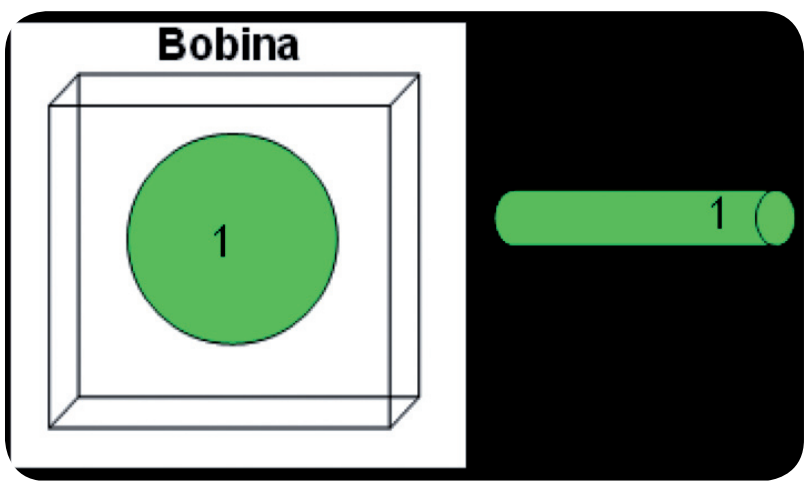

Figura 10. Un receptor, un canal de transmisión para este ejemplo.

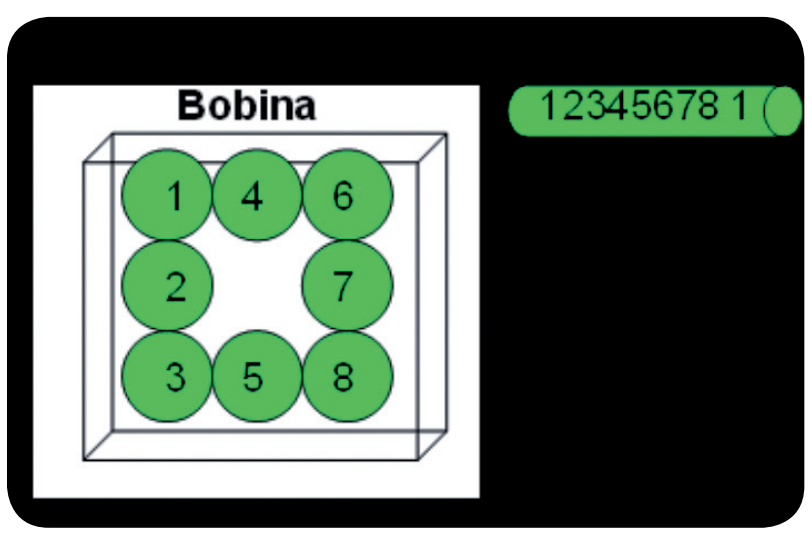

Figura 11. Ocho receptores, un canal de transmisión. El sistema funciona como equipo de un canal para este ejemplo.

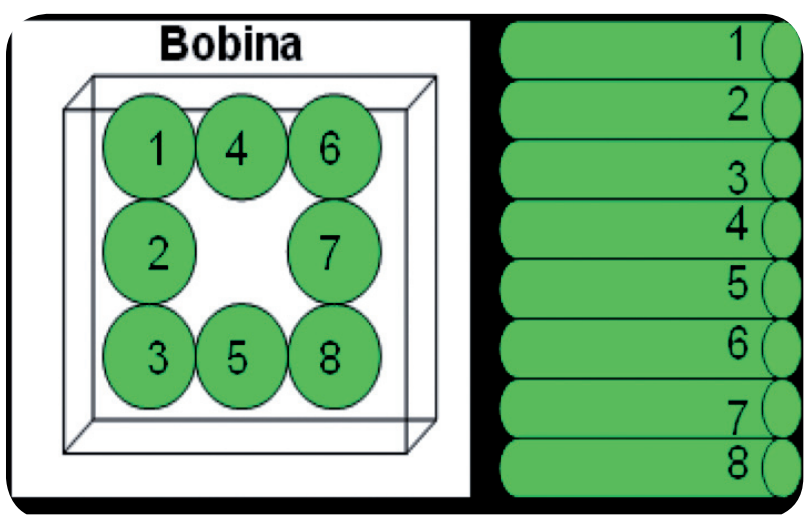

Figura 12. Ocho receptores, ocho canales de transmisión. El sistema funciona como equipo de ocho canales, de forma óptima y máxima utilidad para este ejemplo. 


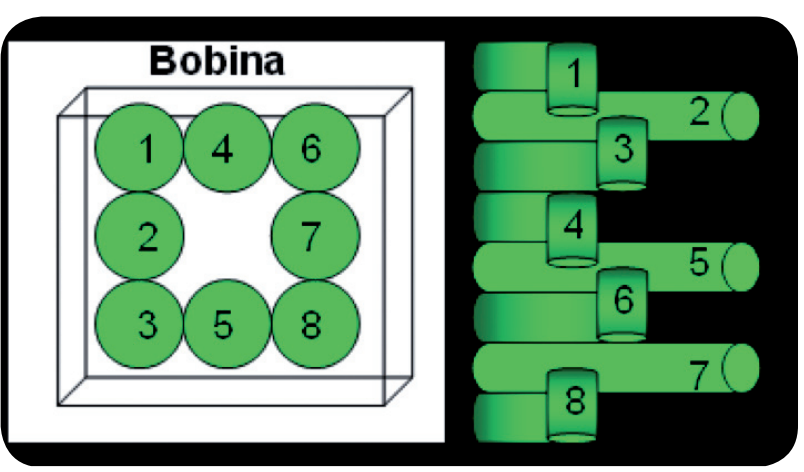

Figura 13. Ocho receptores, tres canales de transmisión 2,5,7. El sistema funciona como equipo de tres canales, los canales $1,3,4,6,8$, son agrupados, para responder a los requerimientos del examen, para este ejemplo.

De acuerdo a lo descrito podemos plantear las siguientes consideraciones generales:

Si tenemos un equipo con 16 canales y bobinas con 8 receptores, el sistema se comportará como un equipo de 8 canales.

Si tenemos un equipo de 4 canales y bobinas de 8 receptores, el sistema se comportará como un equipo de 4 canales.

De acuerdo a lo explicado, un equipo multicanal funcionando en condición óptima, dependiendo de los canales y bobinas multireceptoras utilizadas, puede impactar la agenda de citación de exámenes respecto a su rapidez en un $20 \%$ a $30 \%$. Este dato es sin considerar el uso de adquisición paralela.

\section{EN RESUMEN}

Las tecnologías incorporadas en los últimos años por la RM están pasando frente a nuestros ojos sin una total comprensión y asimilación de sus potencialidades.

La adquisición paralela, los equipos multicanal y los avances en bobinas de elementos múltiples representan el mayor avance tecnológico de la RM.

Una optimización y aplicación integral de todas las técnicas descritas, nos permite realizar estudios con mejor resolución temporal, sin un mayor deterioro de la resolución espacial(8).

Se potencian considerablemente los exámenes que necesitan una evaluación dinámica de la administración de medio de contraste.

La cantidad de señal que proporcionan las bobinas multireceptoras, permiten el uso de matrices altas, espesores de corte y campos de visión pequeños, obteniendo imágenes de muy alta resolución espacial.

Para obtener los mejores resultados, debemos estar conscientes del equipamiento disponible, sus ventajas y limitaciones, lo que sumado a una buena base de fundamentos nos conducirá al mejor rendimiento de los equipos de resonancia magnética.

\section{BIBLIOGRAFÍA}

1. Pruesmann KP, Weiger M. Sensity-Encoded MRI. Medica Mundi. 2000; 44 (2):10-16.

2. Rademaker H., Santa Marta C. Codificación por sensibilidad "SENSE" Velocidad sin límites. Revista Física Médica. 2001;2(2):90-97.

3. van den Brink JS, Watanabe Y, Kuhl CK, Chung T, Muthupillai R, Van Cauteren $\mathrm{M}$, et al. Implications of SENSE MR in routine clinical practice. European Journal of Radiology. 2003; 46(1): 3-27.

4. Ohliger M., Sodickson DK. An introduction to coil array design for parallel MRI. NMR Biomed. 2006; 19(3):300-315.

5. Glockner JF, Hu HH, Stanley DW, Angelos L, King K. Parallel MR imaging: a user's guide. Radiographics. 2005;25(5):1279-1297.

6. Blaimer M, Breuer F, Mueller M, Heidemann RM, Griswold MA, Jakob PM. SMASH, SENSE, PILS, GRAPPA: how to choose the optimal method. Top Magn Reson Imaging. 2004;15(4):223-36

7. Griswold MA, Jakob PM, Heidemann RM, Nittka M, Jellus V, et al. Generalized autocalibrating partially parallel acquisitions (GRAPPA). Magn Reson Med. 2002; 47(6):1202-10

8. van den Brink JS, Watanabe Y, Kuhl CK, Chung T, Muthupillai R, Van Cauteren M,et al. Implications of SENSE MR in routine clinical practice. : Eur J Radiol. 2003; 46(1): 3-27. 